\title{
SCRITTURA E INTERTESTUALITÀ NE $L A$ Cognizione del Dolore di Carlo Emilio GADDA
}

\section{FABRizio RusConi*}

ABSTRACT: L'articolo, prendendo spunto da una espressione di Gianfranco Contini che a proposito di Gadda parla di "rimpianto della letteratura perduta", si ripropone di analizzare, ne La Cognizione del Dolore di Carlo Emilio Gadda, l'emersione di una nostalgia testuale verso quelli autori e quelle opere del passato in cui tra letteratura e mondo non si era ancora frapposto il sospetto, l'incomprensione che caratterizzeranno il XX secolo. Gli spazi, le forme e i modi attraverso cui questa nostalgia si fa sensibile dipendono dal complesso gioco intertestuale affidato al testo. Pastiche, citazione, traduzione sono tra le principali modalità che l'autore della Cognizione sceglie per tematizzare ambiguamente la discontinuità tra presente e passato. Dietro all'apparente sarcasmo e all'ironia con cui sono trattati gli autori catturati nella vertigine intertestuale il lettore potrà avvertire un sentimento di lontananza, rimpianto e nostalgia.

PAROle-ChiAve: Carlo Emilio Gadda; La Cognizione del Dolore; intertestualità; nostalgia; pastiche; traduzione

ABSTRACT: The article is based on an expression of Gianfranco Contini about Gadda, "nostalgia for the lost literature". The intention is to analyze, through La Cognizione del Dolore by Carlo Emilio Gadda, the rise of a textual nostalgia regarding those authors and works of the past in which there wasn't yet the suspicion and incomprehension between the literature and the world that characterize the 20th century. The spaces, forms, and ways in which this nostalgia becomes sensitive depend on the complex intertextual play. The author of Cognizione chooses Pastiche, quotation and translation as the main modalities to ambiguously address the discontinuity between the present and the past. Behind the apparent sarcasm and irony with which the authors caught in the intertextual vertigo are treated, the reader may have a feeling of distance and nostalgia.

KEYWORDS: Carlo Emilio Gadda; La Cognizione del Dolore; intertextuality; nostalgia; pastiche; translation. 



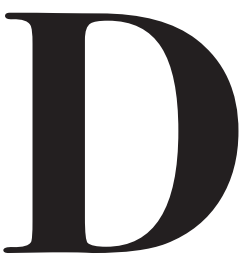

al complesso riprodursi degli spazi citativi delegati al discorso intertestuale e alla sua costante provocazione verso il lettore, che letteralmente si imbatte nelle tracce di altre testualità, dalle più esplicite alle più occulte, dipende una sorta di nostalgia testuale, che tanto nell'opera di Gadda nel suo complesso, quanto nella Cognizione del dolore in particolare si mostra estremamente complesso, contradditorio e sfuggente.

Contini parla del "rimpianto della letteratura perduta" (CONTINI, 1989, p. 12), anche se finisce per spiegarsi questa nostalgia ricorrendo all'autore, alla sua formazione e alla sua psicologia. Questa relazione diretta e causante tra autore e testo toglie valore e forza all'intuizione di Contini, che, risolvendosi nel biografico non trae alla luce tutte le implicazioni che avrebbe se la considerassimo prodotta dalla scrittura e dal suo gioco. Va subito notato che questa "nostalgia della letteratura perduta", come le buone e serie nostalgie, fa oggetto del proprio discorso citante opere e autori consacrati dalla storia della letteratura patria e non solo. Lo stesso Contini è esplicito nell'indicare la preferenza e quindi il rispetto accordata da Gadda per "l'umanesimo e la tradizione". Coglie inoltre un effetto indiretto di questa preferenza che gli fa frequentare nostalgicamente e preferire testi e autori del passato, ossia un tratto di stile che Contini chiama "deformazione manieristica" (CONTINI, 1989, p. 12).

Altra grande intuizione di Contini, l'intertestualità in Gadda non si limita alla citazione di opera e autore ma spesso ne riproduce lo stile, la lingua e il pensiero. Gadda diventa così per Contini uno dei più meravigliosi pasticheur del Novecento, definizione che non può non tener conto di quella "nostalgia della letteratura perduta" che permea così in profondità il testo gaddiano.

Direi proprio che gli esiti parodici e deformanti del pastiche in Gadda non escludono, ma anzi costituiscono semmai la prova della nostalgia verso un passato in cui tra autore, testo e mondo non era ancora intervenuta quella rottura epistemologica che caratterizzerà drammaticamente il Novecento.

Per Bologna il pastiche corrisponde giustamente al tentativo di pervenire a una rappresentazione totale dell'esistente, riproducendo, analiticamente/sinteticamente, la totalità caotica del mondo.

In tal senso, a un Gadda straordinariamente conscio dei propri strumenti di lavoro, non sfuggono le implicazioni di totalità che la tecnica letteraria permette, dato che essa può rappresentare "tutto quanto il caos o il cosmo delle immagini e de' giudizi, dei modi e delle favole, in che si aggroviglia il vivente polipaio della umana comunicativa", come l'autore dichiara in 
un suo saggio dal valore programmatico, Meditazione breve circa il dire e il fare. (GADDA, 1991, p. 445).

È significativo che Gadda colga l'aspetto di mediazione tra mondo e rappresentazione che offre il linguaggio umano. Il pastiche non si volge direttamente al groviglio del mondo nel tentativo di rappresentarlo, sì piuttosto al "vivente polipaio della umana comunicativa", il quale è fatto di "immagini", "giudizi", "modi" e "favole"; ossia esso riproduce forme che sono già simboliche e culturali. Rivolto non al mondo in sé, ma sì ai generi, ai testi che ne danno una rappresentazione simbolica, il pastiche si conferma così essenzialmente intertestuale. Direi che esiste una relazione quasi simbiotica tra la scrittura di Gadda e le scritture del mondo. La nostalgia di Gadda è insomma una nostalgia testuale che guarda a quel cosmo, diventato un caos, in cui "giudizi", "immagini", "modi" e "favole" erano ancora parte di un sistema ordinato nel quale ognuno aveva il suo spazio. Si noti, altresì, che proprio i termini scelti da Gadda hanno una rilevanza estrema e funzionano attivando strati interi di intertestualità ${ }^{1}$. Si prenda, p.e., la parola "immagine": intorno a essa si costruisce tutta una semantica della nostalgia di cui troviamo tracce perfino nella Cognizione.

Così, già il primo verso della poesia Autunno, posta in appendice al romanzo, recita: "Tàcite imagini della tristezza / Dal plàtano al prato!"; concetto ripreso infine nel distico finale: "Tacite imagini e rimota dolcezza/ In ogni novo cuore, per chiari mattini” (GADDA, 1987, p. 499). Sono appunto "imagini della tristezza", nostalgiche per loro natura, che sembrano rivolgersi non tanto ai contenuti della poesia quanto alla sua stessa possibilità di significare, considerando che appunto la poesia vincola il suo potere poetico alle immagini - sono "tacite le imagini" e "rimota" è la "dolcezza" forse proprio perché della poesia non resta che una lontana suggestione, perché le sue immagini si fanno più tenui e opache - non è forse un accenno leopardiano quello alla "collina dolce e brulla" del decimo verso?

E poi lo stesso concetto di immagine - anche nella sua variante poetica e scempia, con una sola " $m$ " - si contamina per effetto dell'influsso di quella poetica dell'ambivalenza che caratterizza i termini principali del vocabolario d'autore's se, infatti, da un lato si relaziona alla poesia, alla stessa possibilità del poetico - che non esisterebbe senza le immagini e il loro potere di suggestione -, e che proprio in tale accezione è attivo nell'intertestualità d'autore ${ }^{3}$; dall'altro, invece, l'imagine (si noti la permanenza ortografica della sola "m", traccia materiale della scrittura in senso derridiano) rimanda alle vuote e ingannevoli parvenze; accezione di cui vi è traccia cospicua ne La cognizione del dolore. In un passaggio testuale tra immagini e parvenza si stabilisce un legame di senso probante:

1 Un discorso analogo potrebbe essere fatto per i termini "giudizi", "modi", "favole", soprattutto quest'ultimo intorno al quale si dispone una fitta intertestualità che attraversa l'intera opera dell'autore, figurando quale concetto autonomo o titolo di opera, si veda Il primo libro delle favole (Neri Pozza, Venezia, 1952). E anche in questo caso la parola coagula intorno a sé accezioni e significati diversi quanto non opposti.

2 Sempre Contini, con grande sagacia scrive a proposito della polivalenza dei termini in Gadda: "La tecnica gaddiana è una forma d'ostinazione: per essa fine è parola ambigua, tra "finalità" e "morte" e pace [di "polemiche e pace"] ambigua, se anche non ci illumina il commento, tra "riposo" e "morte". Tutte queste sono modalità dell'accennata "coagulazione" (CONTINI, 1989, p. 6).

3 Infatti la parola-concetto imagine/i intrattiene una relazione intertestuale che dalla poesia Autunno si riversa in un altro testo importante, "Tendo al mio fine". Vediamo: "Quando si tingerà di oro [il cielo], per il venente autunno, la selva, imagini della tristezza leverà il boreal vento, dal plàtano al prato". Evidentemente questo testo è quasi una parafrasi dell'altro. La scrittura ripete nella differenza, ripete variando; semina tracce intertestuali e intratestuali che il lettore attento deve riconoscere. 
Cogliere il bacio bugiardo della Parvenza, coricarsi con lei sullo strame, respirare il suo fiato, bevere giù dentro l'anima il suo rutto e il suo lezzo di meretrice. $\mathrm{O}$ invece attuffarla nella rancura e nello spregio come in una pozza di scrementi, negare, negare [...] Ma l'andare nella rancura è sterile passo, negare vane immagini, le più volte, significa negare se medesimo (GADDA, 1987, p. 354, corsivo nostro).

Esiste continuità tra il "bacio bugiardo della Parvenza" e le "vane immagini". Siamo evidentemente di fronte a un aut/aut. O negare per andare verso la conoscenza autentica, verso la vera ma dolorosa cognizione - un legame di senso si stabilisce tra questo passaggio e il titolo del romanzo - correndo il rischio di riconoscere anche se stessi come il prodotto di false immagini. Oppure adagiarsi comodamente nella "bugia" delle vane immagini accettando il bacio della Parvenza e il suo inganno felice. Si badi che abitare nell'inganno dell'immagine significa anche accettarne i miti e le favole, anche quelle che attengono alla letteratura. In un passaggio che ha una relazione evidente con il tema della nostalgia del testo si legge:

L'imagine tradizionale e ab eterno romantica dello scrittore-creatore, dell'ingegnoso demiurgo, che cava da sé liberamente la libera splendidezza dell'opera e nei liberi modi di un suo stile ne propaga foco alle genti, porgendo in una e rara occasione di esercizio al tartufare arguto dei critici e novo incentivo a sventolare a tutte le bandiere della patria, e de' turriti municipi, è imagine in sul nascere viziata" (GADDA, 1991, p. 427-428).

È allora evidente che o si vive nell'inganno, adagiandosi comodamente nell'immagine tradizionale e romantica dello "scrittore-creatore", geniale, libero e engagé, o nella cognizione fratturata e postuma, direi post-romantica, di chi sa di occupare una posizione di irrilevanza e perfino di isolamento, facendo della propria un'esistenza infelice o quantomeno nostalgica: dal lato infelice si ha Gonzalo o una delle altre maschere biografiche di Gadda, da quello felice scrittori come il vate Caçoncellos che vivono risibilmente nell'illusione e nell'inganno.

Ma attenzione: non si vuole qui sostenere che il gioco della scrittura sia sempre trasparente o privo di ambiguità, né che ogni dichiarazione, asserzione consegnata alla scrittura debba essere presa alla lettera. Pervasa dal gioco sottile dell'ambivalenza la scrittura può dar vita a una parodossale coincidencia oppositorum attraverso cui, ciò che è letteralmente dichiarato, è negato in un altro piano di lettura. In tal senso le forme di parodizzazione manzoniana, così abbondanti nella Cognizione, possono essere interpretate seriamente come nostalgiche rispetto a quell'autore, Manzoni, e a quel testo, I promessi sposi. Precisiamo: la nostalgia non è quella di Gadda per Manzoni, nonostante questa paternità sia stata chiamata spesso in causa per spiegare i testi gaddiani ${ }^{4}$, è invece e piuttosto una nostalgia che si costruisce nel testo, a partire dal testo e che lascia tracce ambigue e provocazioni.

4 E pure Contini chiude il discorso ricorrendo al biografico: "Il culto di Gadda per Manzoni che durò fino agli ultimi giorni, quando amici si davano il cambio a leggergli il gran romanzo [I promessi sposi, N.d.R.], potrebbe a prima vista sembrare paradossale, se non fosse che il divorato dalla nevrastenia percepisce, tenebrando le pagine del suo impavido collega (e senza neppure varcare il perimetro dei Promessi sposi), i toni d'una "tragica sinfonia" (CONTINI, 1989, p. 74). 
Lo si è visto concretamente, il testo della Cognizione sembra dialogare di preferenza con gli autori e le opere dei secoli aurei della letteratura, da Orazio a Shakespeare, da Platone a Virgilio, da Heine a Manzoni. Ma appunto, soprattutto Manzoni, la cui figura e la cui opera possono fare le veci di una paternità intertestuale quasi perfetta. Occultato, come si è visto, a ogni livello testuale, il grande scrittore lombardo esemplifica quel meccanismo che, mentre corrode con lo strumento della parodia citativa, incaricandosi insomma di decostruire testo, contesto e autore, dall'altro verso istituisce un vero e proprio confronto nostalgico con questa fonte.

Il confronto nostalgico con Manzoni si dà nel testo, spesso occultato spesso provocato. Nella Cognizione, il sottotesto manzoniano pervade infatti lo stesso cronotopo romanzesco. Come si è già osservato, i luoghi geografici del romanzo di Gadda sono una riscrittura parodica di quelli manzoniani. Così "il Serruchón è qualcosa di simile, per il nome e più per l'aspetto al manzoniano Resegone" (CONTINI, 1989, p. 16). Ciò che si viene istituendo è una sovrapposizione straniante tra il Maradagàl e la Brianza, regione connotata tanto da elementi biografici quanto letterari: è qui, infatti, tra Como e Lecco che il romanzo manzoniano ha luogo; ma è sempre qui, in questa regione, che il padre di Gadda fece erigere la villa di famiglia. D'altronde la stessa scelta della lingua maradagalese/castigliana può essere letta come una suggestione manzoniana. Quella che ne I promessi sposi era semplicemente la lingua dei dominatori, acquista ne La cognizione un peso autonomo. L'operazione romanzesca gaddiana deterritorizalizza e capovolge i dati di partenza manzoniani. La Brianza, la cui geografia si riconosce nelle numerose descrizioni di un lontano Maradagal, funziona nostalgicamente tanto rispetto a un testo quanto rispetto a una geografia esistenziale.

Ugualmente la scelta di una lingua straniera, il Maradagalese/castigliano segnala nostalgicamente la distanza da una lingua, "l'italiano", che è unicamente una lingua letteraria (e già per questo intertestuale), sempre tradotta, e esistente di fatto solo nelle sue varietà regionali o dialettali - prova è che di "italiano" non si parla nella Cognizione, bensì di napoletano, di "dialetti del Serruchòn" dalla "cadenza ossitona" (GADDA, 1987, p. 126) ossia propriamente nordici, di "dialetti celtici" (GADDA, 1987, p. 389), o infine di strani ibridi linguistici come quel "dialetto turcasso-celtico della Keltiké" (GADDA, 1987, p. 394). Direi, anzi, che questo confronto nostalgico, segnato da una distanza culturale e linguistica, è fattore marcante dell'intertestualità in Gadda. Non solo Manzoni e il suo mondo linguisticamente aureo-toscano, lingua topograficamente e socialmente di "grado-zero" (CONTINI, 1989, p.17), ma anche Platone, Virgilio, Orazio, Shakespeare e Heine, Cervantes: ognuno di questi autori-fonti segnala una distanza linguistica e culturale incolmabile. Il greco antico, il latino classico, l'inglese elisabettiano e lo spagnolo cervantesco o manzoniano, funzionano come varietà, come dialetti, all'interno di quella grande madre, o lingua comune che è la letteratura.

Dentro queste coordinate, la traduzione può assolvere funzioni ambivalenti. Attraverso la traduzione si palesa il tentativo di colmare la distanza, annullando con un unico atto di volontà la differenza linguistico-culturale e storica. Ma più spesso ne La cognizione del dolore la traduzione è a carico del lettore che, come si è visto, deve riconoscere la fonte e tradurne la 
lingua straniera. Lo stesso Maradagalese, che è lingua letteraria poiché lingua di scrittura, si insinua nel testo provocando il lettore a farsi traduttore, sperimentando sempre un certo straniamento che è, a suo modo, una forma di nostalgia. Si apre qui il grande capitolo del Gadda traduttore. Le opzioni possibili a questo punto sono varie e perfino antitetiche. La più frequente, come si è osservato, è la non-traduzione: opzione quasi obbligata quando il gioco testuale ruota intorno alle citazioni. Solo così si conserva la differenza e la riconoscibilità di ciò che è estraneo. Altrove però Gadda è chiamato a cimentarsi in quella modalitá integrale di intertestualità che è la traduzione vera e propria. In questo caso, scartata, per ovvie ragioni, la prima opzione, nel caso riscrittura paradossale e borgesiana, resta percorribile a Gadda una traduzione-limite, nelle parole di Contini: "il caso-limite, di una certa possibilità di traduzione, un fatto nella storia delle traduzioni probabilmente senza precedenti - di sommo interesse anche teorico" (1989, p. 55). Quella di Gadda sarebbe insomma una traduzione che viola il dogma traduttorio del dire quasi la stessa cosa, muovendo verso esiti di espressionismo o di macaronico (SEGRE, 1979, p. 176).

Paradigmatiche sono proprio le scelte di traduzione, compiute su autori che alla differenza linguistica sommano una distanza culturale e diacronica. La nostalgia del testo non può non passare anche da questi autori lontani per lingua e cultura. Così,

Per il volume antologico Narratori spagnoli. Raccolta di romanzi e racconti dalle origini ai nostri giorni, a cura di Carlo Bo, pubblicato nel 1941, Gadda traduce, infatti, la Peregrinación sabia ("Il viaggio di saggezza") di Alonso Jerónino de Salas Barbadillo e El mundo por de dentro ("Il mondo com'è") di Francisco de Quevedo y Villegas (CHECOLA, 2014, p. 190).

E ancora: "Giunge infine la proposta di Vittorini di tradurre Achim von Arnim per il Teatro tedesco" (CHECOLA, 2014, p. 191). Gadda avrebbe dovuto occuparsi dell'opera “Die Appelmänner, inclusa nel progetto del Teatro tedesco, [...] pubblicata dallo scrittore nel 1813 nel suo volume di scritti teatrali, intitolato Schaubühne" (CHECOLA, 2014, p. 185), ma purtroppo non consegnò mai la traduzione completa. Tanto i due autori spagnoli quanto il tedesco sembrano continuare, nell'ambito della traduzione, quel dialogo, fatto di lontananza e nostalgia, di cui si hanno cospicue tracce intertestuali ne La cognizione del dolore. La strada percorsa da Gadda per abbattere questa distanza sarà in tutti questi casi quella di una traduzione "espressionista" in cui si dà una mescolanza tra antico e nuovo, tra le più "varie tradizioni di linguaggio, dall'aulica alla dialettale" (CONTINI, 1989, p. 56).

Per concludere, la nostalgia del testo per "la letteratura perduta" si fa tangibile nel gioco parodico e manipolatorio della citazione di testi e autori che provocano straniamento in virtù della differenza culturale e linguistica; ma anche nelle scelte di traduzione che più hanno stimolato al cimento l'autore della Cognizione. Scrittori barocchi come Barbadillo o Quevedo, o del romanticismo tedesco, come Arnim, sembrano in grado di risvegliare una nostalgia per un tempo in cui tra autore, opera e personaggi non si era ancora insinuato il reciproco sospetto. Concordia perfetta che conferiva alla letteratura il suo posto nel mondo. 


\section{Referências}

BOLOGNA, C. Tradizione e fortuna dei classici italiani. II. Dall'Arcadia al Novecento. Torino: Einaudi, 1994.

CHECOLA, A. Una traduzione inedita di Gadda: "gli Appelmänner" di Achim Von Armin. In: MARCHI, M.; VELA, C. (orgs.). Meraviglie di Gadda. Seminario di studi sulle carte dello scrittore. Pisa: Pacini Editore, 2014, p. 185-201.

COMPAGNON, A. O trabalho da citação. Trad. Cleonice Mourão. Belo Horizonte: Editora UFMG, 1996.

CONTINI, G. Quarant'anni di amicizia. Scritti su Carlo Emilio Gadda (1934-1988). Torino: Einaudi, 1989.

GADDA, C. E. La cognizione del dolore. Edizione critica a cura di Emilio Manzotti. Milano: Einaudi, 1987.

1991, p. 427-670. I viaggi e la morte. In: Saggi, giornali, favole e altri scritti. Milano: Garzanti,

HEINE, H. Das Buch der Lieder. Trad. F. Amoroso. Pisa: Seu, 1996.

MANZONI, A. I promessi sposi. Milano: Mondadori, 1995.

MANZOTTI, E. Nota al testo. In: GADDA, C. E. La cognizione del dolore. Milano: Garzanti, 2011, p. 206-401.

SEGRE, C. Semiotica filologica. Testo e modelli culturali. Torino: Einaudi, 1979.

Recebido: 10/06/2018

Aprovado: 18/09/2018 